\title{
Qualitative and Quantitative Study of Gukefuyao Preparation Using FT-IR and HPLC
}

\author{
Jingbo SUN ${ }^{1, a^{*}}$, Lihua Zhang ${ }^{1, b}$, Zitong Li ${ }^{1, c}$, Chunying Qiao ${ }^{1, d}$, Li Qu ${ }^{2, \text { e }}$ \\ ${ }^{1}$ Pharmaceutical college of Beihua University, Jilin, China \\ ${ }^{2}$ Beihua School of Public Health, Jilin, China \\ a sjb781219@163.com, b1592123431@qq.com, '957918240@qq.com, ${ }^{d} 1744935552 @ q q . c o m$, \\ e362805812@qq.com
}

\begin{abstract}
Keywords: Gukefuyao, Fourier transform infrared spectroscopy, imperatorin, HPLC
Abstract. Objective To establish methods for the quantitative and qualitative identify of Gukefuyao preparation. Methods Characteristic absorption peaks of Gukefuyao preparation powder and its different extracts were identified, compared and analyzed according to the peaks' shape, position and intensity in infrared spectra. The content of imperatorin was determined by HPLC. Results Fourier transform infrared spectroscopy (FT-IR) Fingerprint of 9 main common peaks was established preliminarily. The mobile phase was acetonitrile-water(62:38) with a flow rate at $1.0 \mathrm{~mL} / \mathrm{min}$. Detection wavelength was $254 \mathrm{~nm}$. The linear ranges for imperatorin was $0.065 \sim 1.032 \mu \mathrm{g}$. The average recovery was $99.3 \%(n=6)$ and RSD was $3.3 \%$. In the meantime, the content of imperatorin in Gukefuyao should not less than $0.05 \mathrm{mg} / \mathrm{g}$. Conclusion The methods are simple, rapid and accurate, and can be used for the qualitative and quantitative analysis of Gukefuyao preparation.
\end{abstract}

\section{Introduction}

Gukefuyao is a kind of traditional Chinese medicine (TCM) powders for external use. It can be well used for the treatment of traumatic injury in which the parts of the body becomes red, swelling, hot, pain and soft tissue adhesions. The preparation has been used for more than ten years in Jilin Longtang Chinese medicine hospital and unanimously approved by the patients. Gukefuyao is only an agreement prescription(home preparation).Its original quality standard is too simple and can not to show its real quality. Therefore, the paper was to carry out a series of studies on the identification of Gukefuyao.

Infrared spectroscopy is a kind of identification method, which has been reported in the research of traditional Chinese medicine and its preparation in recent years [1-4]. Therefore, infrared spectroscopy was used for the identification and analysis fo Gukefuyao and its different solvent extracts. Angelica dahurica is one of the main medicines in the preparation, and imperatorin is one of the main active ingredients of Angelica dahurica. By using imperatorin as screening index component, the high-performance liquid phase chromatography (HPLC) method was established for the content determination of Gukefuyao. It is very important for the study to improve the current quality standards of Gukefuyao and provided scientific basis for its quality control method.

\section{Instrument and reagent}

Instrument. Burker TENSOR27 Fourier transform infrared spectrometer (Bruker, German), Agilent High-performance Liquid Chromatography (Agilent, America).

Reference substance. Imperatorin (batch lot: 11826-200410, specification: 20mg, for the content determination) provided by the National Institutes for Food and Drug Control.

Reagent. Acetonitrile and methanol are chromatographic pure reagent. Potassium bromide is spectrum pure reagent. Other reagents are the analysis pure. Water is Wahaha Pure water.

Gukefuyao is provided by Jilin Longtang Chinese medicine hospital. 


\section{Methods and results of IR identification of Gukefuyao}

Preparation of the sample. 3 samples of Gukefuyao (about $3 \mathrm{~g}$ per sample) were weighted and then ultrasonic extracted with $30 \mathrm{~mL}$ of ethanol, acetone and ethyl acetate for $20 \mathrm{~min}$, respectively. After extraction, the filtrate is concentrated and dried in the oven at $60{ }^{\circ} \mathrm{C}$ for $6 \mathrm{~h}$, then placed in the dryer for later use.

IR spectroscopy determination of Gukefuyao. Gukefuyao powder and 3 kinds of its extracts (2.1) were separately mixed with the amount of potassium bromide, grinded homogeneity and then tabletting. The IR spectroscopy of these four samples were determined. The results are shown in figure 1and table 1 .

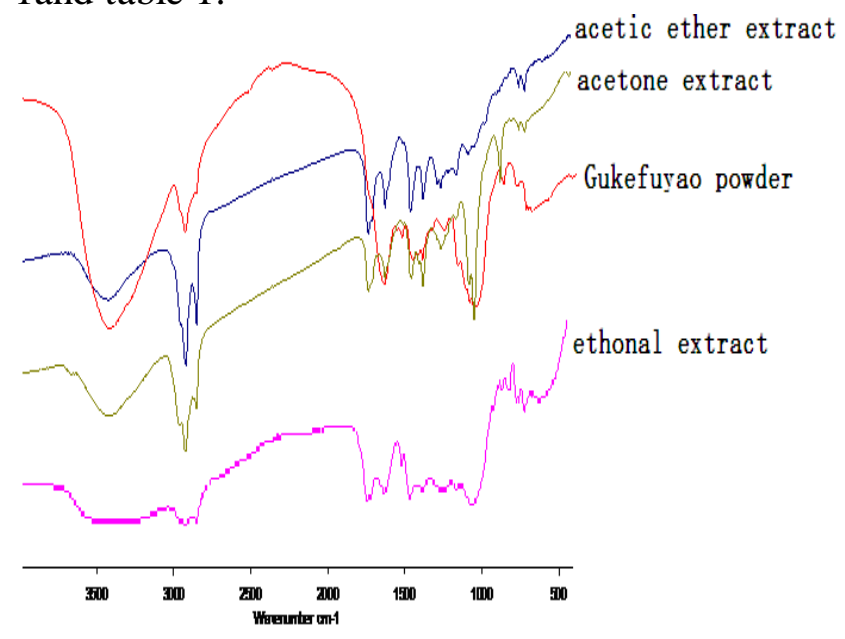

\begin{tabular}{ccccc}
\hline $\begin{array}{c}\text { No } \\
.\end{array}$ & \multicolumn{4}{c}{ Peaks $\left[\mathrm{cm}^{-1}\right]$} \\
\hline & $\begin{array}{c}\text { powde } \\
\text { r }\end{array}$ & $\begin{array}{c}\text { EtO } \\
\text { H }\end{array}$ & Acetone & EtOAc \\
\hline 1 & 3418 & 3393 & 3418 & 3424 \\
2 & 2926 & 2926 & 2926 & 2924 \\
3 & 2855 & 2853 & 2855 & 2853 \\
4 & 1632 & 1628 & 1628 & 1628 \\
5 & 1450 & 1464 & 1456 & 1464 \\
6 & 1385 & 1379 & 1383 & 1381 \\
7 & 1080 & 1072 & 1080 & 1088 \\
8 & 768 & 760 & 760 & 760 \\
9 & 712 & 721 & 721 & 721 \\
\hline
\end{tabular}

Fig. 1 FTIR fingerprints of Gukefuyao powder and their extract

Tab 1 FTIR fingerprint of characteristic absorption peaks of Gukefuyao powder and their extracts

Figure 1 and Table 1 shows that 9 common peaks were existed both in the IR spectra of Gukefuyao powder and in the IR spectra of its 3 kinds of extracts. This means that the 9 peaks are related to the main active components and can not change with the different solvent, and then determined to be the characteristic peaks of Gukefuyao. Among of them, peak of $3410 \mathrm{~cm}^{-1}$ is hydroxyl absorption peak, peaks of $2925 \mathrm{~cm}^{-1}$ and $1380 \mathrm{~cm}^{-1}$ are $-\mathrm{CH}_{2}$ - and $-\mathrm{CH}_{3}[5]$.

\section{Content Determination Methods and Results of Gukefuyao}

Chromatographic Conditions. The chromatographic conditions were a column C18 (Diamonsil $250 \mathrm{~mm} \times 4.6 \mathrm{~mm}, 5 \mu \mathrm{m}$ Analytical Column, America). The mobile phase was acetonitrile- water (62:38) and the flow rate was $1.0 \mathrm{~mL} / \mathrm{min}$. The operating temperatures were maintained at room temperature but the oven was heated at $25^{\circ} \mathrm{C}$ to favour the mobile phase flow through the column. The detection wavelength UV was $254 \mathrm{~nm}$ and the injection volume was $10 \mu \mathrm{L}$. The number of theory plates was not less than 3000 determined by the peaks of imperatorin.

Reference solution. To accurately weigh $1.29 \mathrm{mg}$ of imperatorin to $10 \mathrm{ml}$ volumetric flask and add $5 \mathrm{ml}$ of methanol. After that, the volumetric flask were shaken vigorously to promote the solubilization of imperatorin, then made up to the mark with methanol and were stored at $-4^{\circ} \mathrm{C}$ for measurement.

Sample solution. About $1 \mathrm{~g}$ Gukefuyao powder was accurately weighted and soaked in $45 \mathrm{ml}$ methanol, then extracted by means of ultrasonic extraction $(300 \mathrm{~W}, 50 \mathrm{kHz})$ for 1 hour. After cool to the room temperature, add methanol to the mark and then filtrated with $0.22 \mu \mathrm{m}$ millipore membrane for HPLC analysis. 
Negative sample solution. The negative sample was prepared according to the proportion of Gukefuyao prescriptions except Radix Angelicae dahuricae. The preparation of the negative sample was the same as "sample preparation".

Standard curve. The peak areas of $0.5 \mu l, 1 \mu \mathrm{l}, 2 \mu \mathrm{l}, 4 \mu \mathrm{l}$ and $8 \mu \mathrm{l}$ imperatorin reference solution were measured, respectively. The regression equation and the correlation coefficient were established as: $\mathrm{Y}=64559 \mathrm{X}-5.9709, \mathrm{r}=0.9997$, where $\mathrm{Y}$ is the peak area and $\mathrm{X}$ is the injection volume of imperatorin $(\mu \mathrm{l})$. The experimental results show that the peak area had a good linear relationship with the amount of imperatorin in the range $0.065 \sim 1.032 \mu \mathrm{g}$.

Specific experiments. The specificity of the method was verified by comparing the chromatograms of standard imperatorin, Gukefuyao sample and its negative sample with no Radix Angelicae dahuricae. The chromatograms obtained in HPLC for the negative sample did not show the peak with a similar retention time to that of the imperatorin(9.770 $\min \pm 10 \%$ ) (Figure 2). So, it was observed the absence of interferences of the other pharmaceuticals for Gukefuyao preparation, because none of the peaks appears at the same retention time as imperatorin peak. The results shows that the developed method is selective in relation to the other pharmaceuticals in the Gukefuyao preparation.

Stability experiments. The peak areas of the same sample solution was determined by HPLC at different time (at $0 \mathrm{~h}, 2 \mathrm{~h}, 4 \mathrm{~h}, 6 \mathrm{~h}, 8 \mathrm{~h}, 10 \mathrm{~h}$ and $12 \mathrm{~h}$, respectively) and the relative standard deviation was $1.52 \%$. The results indicated that the tested sample solution was kept stable within 12 hours.

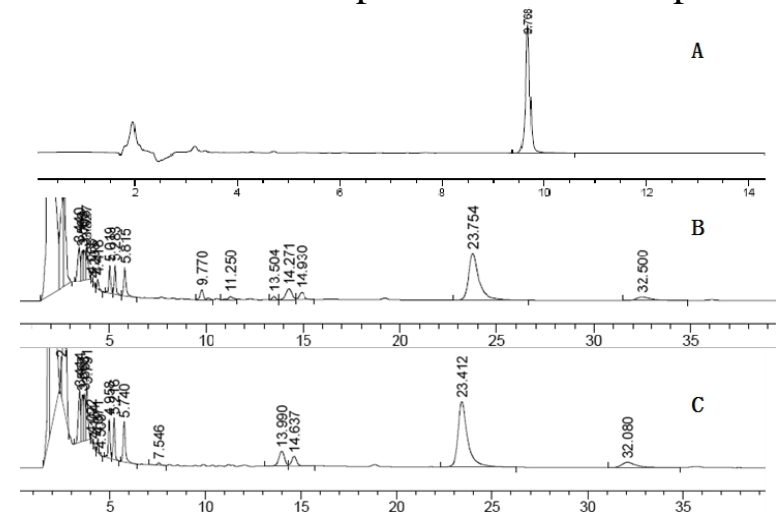

Fig 2 Chromatograms of imperatorin(A), sample (B) and blank sample (C)

Precision experiment. The precision of the method was assessed by testing the peak areas of one standard solutions 6 times. Then the average peak area and percentage of relative standard deviation were obtained as 148.56 and $\mathrm{RSD}=0.69 \%$, respectively. The results show that the HPLC had a good precision. In the case of the developed method, precision has been validated for various repeatability studies.

Repeatability experiment. 6 samples from one batch of Gukefuyao were prepared according to the method of "sample solution". The imperatorin peak areas of the 6 samples were measured and then caculated the imperatorin content of each samples and RSD (1.3\%). The results showed that the method has a favorable repeatability. Therefor, the study was considered validated.

Recovery experiment. The recovery experiment by adding standard samples was carried out for nagetive Gukefuyao samples with addition of three-level imperatorin. The average amount of imperatorin recovered in relation to the added amount was calculated as $99.3 \%$ (RSD 3.3\%). The analytical results of six samples show that the method has a good recoveries.

Determination of imperatorin in Gukefuyao. The concentration levels of imperatorin in 3 lots of Gukefuyao(lot number: 0506, 0507, 0508) were mesured and the results were 0.0605, 0.0613 and $0.0598 \mathrm{mg} / \mathrm{g}$. For the analytical procedure of Gukefuyao sample, the methods were the same as described previously. According to the results, the content of imperatorin in Gukefuyao shall not be less than $0.05 \mathrm{mg} / \mathrm{g}$. 


\section{Discussion}

Traditional Chinese Medicine (TCM) is the precious wealth of Chinese nation, and has made a great contribution to the healthcare of Chinese people and the prosperity of the Chinese nation. TCM has attracted more and more attention for its significant biological activity and the world wide demand for the TCM has provided a broad space for its development. While the quality control becomes one of the most important bottlenecks in the development of TCM and its prscriptions. Among of the analysis and identification methods, infrared spectrometry has an important significance and practical values in the qualitative identification and content determination of TCM and preparation for its high efficiency, convenience and environmental protection[1].

FT-IR was used to identified Gukefuyao powder and its different solvent extracts in the paper. Little difference found in those infrared spectras showed that the main absorption peaks were consistent and could not be affected by extraction solvent. Therefore, 9 main peaks of Gukefuyao in these IR spectra were attributed as the infrared characteristic absorption peak.

Imperatorin from sovereign drug Angelica dahurica of Gukefuyao has many important functions such as antibacterial, anti-inflammatory, antipyretic and analgesic effects[6-7]. So the quantitatively experiment of Gukefuyao was designed with the content of imperatorin as index. The determination wavelength of imperatorin were selected as 300nm [8], 254nm [9-10] or $248 \mathrm{~mm}$ [11] in some other papers. On the basis of above mentioned contents, UV wavelength scanning of imperatorin was obtained and the result showed that the maximum absorption wavelength of imperatorin was at about $249 \mathrm{~nm}$. As a result, the detection wavelength was set at $254 \mathrm{~nm}$ for facilitate the detection and popularization.

This paper develops the qualitative and quantitative analysis methods for evaluating the quality of Gukefuyao. These methods are simple, stable and accurate and can be used for the quality control of Gukefuyao.

\section{References}

[1] H ZHAN, MT LIU, J FANG, MH FU. Application of near infrared analytical technique in identification and content determination of traditional Chinese medicing, Chin J Exp Tradit Med Form. 12 (2015) 231-234.

[2] ZG W, H D, JH W, et al. To quickly identify Cortex lycii and its adulterant Jasminum giraldii Diels using NIR, China's tradit Chin Med Inform, 15(2011)224-226.

[3] Y B, YY L. To analyze Yiqing granule produced by different manufactures using NIR spectrometry, Chin J Chin Mater Med, 20(2008), 2413-2416.

[4] DP T, DQ Z, LP L, et al. A Fourier transform infrared spectrum method for rapid identification of traditional Chinese medicine particle prescription, ChP. Patent 201410595431.7.( 2014).

[5] SJ S, Q Z, J L, et al. Study on the identification of standard and false Banxia by two-demensional infrared correation spectroscopy, Spectroscopy and Spectral Analysis, 4(2004), 427-430.

[6] YY W, GH J, YY M, et al. Pharmacological activities research progress of angelica dahurica, Lishizhen Med Mater Med Res, 3(2009), 625-627.

[7] YW W. The chemical composition, pharmacological function and research progress of angelica dahurica, Chin J ethnomedicine and ethnopharmacy, 17(2011), 28-29.

[8] HZ W, HF L, J Z, et al. Determination of imperatorin and isoimperatorin in Yuan Hu Zhi Tong Pian by HPLC method, Chin Tradit Herbal Drugs, 7 (2007) , 1018-1019.

[9] HW X, Q M, Q L, et al. Simultaneous Determination of Ferulic Acid, 5-Methoxypsoralen, Imperatorin and Isoimperatorin in Duliang Dropping Pills by High Performance Liquid Chromatography, Chin J Exp Tradit Med Form, , 16(2011), 81-85.

[10] HJ H, ZH L, GY L, et al. Determination of Imperatorin and Isoimperatorin in Angelicae Dahuricae from Different Sources by HPLC, J Zhejiang University of Tradit Chin Med, 3(2010), 418-420. 
[11] XC C, YR W, SP L, et al. Study on the extraction technology of Radix Angelicae dahuricae, Chin Tradit Patent Med, 2(2005), 145-147. 